\title{
Mehr Lebensqualität
}

\author{
Wolfgang Bayer
}

\begin{abstract}
Menschen mit Behinderung können mit dem Persönlichen Budget ihr Leben besser gestalten. Für die Anbieter sozialer Dienste und Einrichtungen ist die neue Leistungsform eine Chance, alle ihre Angebote darauf hin zu überprüfen, ob und wie sie individuelle Teilhabe ihrer Nutzerinnen und Nutzer fördern.
\end{abstract}

Nutzerinnen und Nutzer sind sich sicher: Das Persönliche Budget ist geeignet, sie bei der Realisierung neuer Teilhabechancen und bei der Verbesserung ihrer Lebensqualität zu unterstützen. Sie äußern sich mit dem Persönlichen Budget zufrieden und berichten darüber hinaus von einem durch die selbstbestimmte Wahl der Unterstützung gestiegenem Selbstbewusstsein. (1) Dies ist sicherlich eines der wichtigsten Ergebnisse des Bundesmodellprojektes zum Trägerübergreifenden Persönlichen Budget.

Das seit dem 1. Januar 2008 als Rechtsanspruch bestehende Persönliche Budget war von 2004 bis 2007 in einem bundesweiten Modellprojekt in insgesamt 14 Regionen erprobt und beforscht worden. Die nun vorliegenden abschließenden Berichte und Gutachten (2) lassen im Hinblick auf die Wirksamkeit, aber auch im Hinblick auf die noch bestehenden Herausforderungen klare Schlüsse zu, trotz der relativ geringen Zahl von 847 Persönlichen Budgets (494 in den Modellregionen). Die geringe Gesamtzahl hatte sicherlich in der anfänglich vorhandenen Unsicherheit über Form, Inhalte und Wege der Bewilligung eine Ursache. Auch die anfänglich zu lange Bearbeitungsdauer förderte zunächst nicht das Interesse an der Mitwirkung und schließlich trug auch die zum Teil rigide Nachweisverpflichtung zur vorhandenen Zurückhaltung bei. Im Verlauf der dreijährigen Projektphase steigerte sich mit zunehmender positiver Erfahrung die Zahl der Neubeantragungen von Quartal zu Quartal, was Ausdruck für die entstandene Dynamik ist, die es weiter zu nutzen gilt.

Nach Auswertung der Projektphase bleiben insbesondere Herausforderungen bestehen im Hinblick auf

- den sozialrechtlichen Rahmen

- die zum Teil sehr unflexible Handhabung des Persönlichen Budgets durch Sozialleistungsträger

- die unzureichende Finanzierung einer nutzerorientierten Budgetberatung und Budgetassistenz

- die Verbesserung der Zusammenarbeit der Leistungsträger, um eine Träger übergreifende Hilfegewährung zu fördern

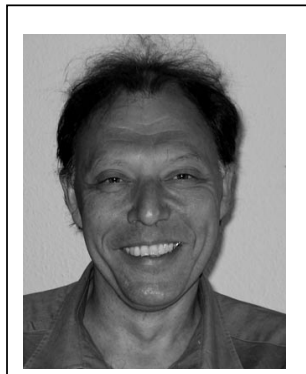

Wolfgang Bayer ist Diplom-
Pädagoge mit Ausbildung zum
Social Manager und seit 23 Jah-
ren in der Psychiatrie sowie in
der Fortbildung tätig. Er leitet
den Einrichtungsverbund Psy-
chiatrie Süd im Stiftungsbereich
Integrationshilfen der v. Bodel-
schwinghschen Anstalten Bethel in Bielefeld und befasst sich mit den Themen Enthospitalisierung und De-Institutionalisierung, Aufbau gemeindeintegrierter Unterstützung, Vernetzung ambulanter und stationärer Hilfen, individuelle Hilfeplanung und Stärkung von Nutzerinnen und Nutzern. Beim Bundesverband evangelische Behindertenhilfe e.V. (BeB) gestaltet er Arbeitsgruppen zu sozialrechtlichen und psychiatrischen Themen mit und leitete zuletzt die Arbeitsgruppe zur weiteren Entwicklung des Persönlichen Budgets nach Abschluss des Bundesmodellprojekts. In diesem $\mathrm{Zu}$ sammenhang hatte er die Federführung bei der Erarbeitung des aktuellen Diskussionspapiers des Verbandes zum Persönlichen Budget (Internet http://www.bebnet.de/files/download/pdf/ 2008/sonstige/2008-04-12DiskussionspapierPBGL6.pdf.)

E-Mailwolfgang.bayer@bethel.de

- eine stärkere Nutzung des Persönlichen Budgets für den Bereich Teilhabe am Arbeitsleben und schließlich

- die Nutzung des Selbstbestimmung und Individualität fördernden Impulses des Persönlichen Budgets auch für die Bereiche der bisherigen Sachleistungen.

\section{Spezifische individuelle Wirkungen des Persönlichen Budgets}

Zur Erinnerung: Die bereits im Jahre 2001 im SGB IX formulierte Ausrichtung des Sozialrechtes und die im Jahre 2006 von den Vereinten Nationen verabschiedete und im Mai 2008 in Kraft getretene UN-Konvention (3) legen das Ziel der vollen und vor allem wirksamen Teilhabe an der Gesellschaft fest. Aber auch Fachverbände wie der Bundesverband evangelische Behindertenhilfe (BeB) haben in ihren Grundsatzpapieren darauf hingewiesen, dass 


\section{Anmerkungen}

(1) Wissenschaftliche Begleitforschung 2007a: Wissenschaftliche Begleitforschung zur Umsetzung des Neunten Buches Sozialgesetzbuch (SGB IX) - Rehabilitation und Teilhabe behinderter Menschen - Begleitung und Auswertung der Erprobung trägerübergreifender Persönlicher Budgets. Abschlussbericht Juli 2007, S.36; Internet http://www.bmas.de/coremedia/generator/23072/property=pdf/f366_forschungsbericht.pdf.

(2) Wissenschaftliche Begleitforschung 2007a; Welti, Felix; Rummel, Kerstin (2007): Rechtsfragen des Persönlichen Budgets nach § 17 SGB IX - Gutachten im Rahmen der wissenschaftlichen Begleitung der modellhaften Erprobung Persönlicher Budgets nach § 17 Abs. 6 SGB IX; Internet http://www.bmas.de/ coremedia/generator $/ 23074 /$ property $=$ pdf/f367_forschungsbericht_anlage_zu+ f366.pdf. Ramboll Management GmbH (2007): Expertise zu Verwaltungsverfahren beim trägerübergreifenden Persönlichen Budget; Internet http://www.bmas.de/ coremedia/generator/23076/property=pdf/f368_forschungsbericht_anlage_zu__f366.pdf.

(3) Vereinte Nationen 2006: Übereinkommen über die Rechte von Menschen mit Behinderung, amtliche deutschsprachige Übersetzung; Internet http://www.bmas.de/coremedia/ generator/2888/property=pdf/uebereinkommen_ueber_die_rec hte__behinderter_menschen.pdf.

(4) Bundesverband evangelische Behindertenhilfe (BeB) (2008a): Auf dem Weg zur Inklusion. Die Weiterentwicklung der Eingliederungshilfe (Kurzfassung), S. 4; Internet http://www.beb-ev.de/ files/pdf/2008/sonstige/2008-02-26Inklusion_Kurzfassung.pdf.

(5) Wissenschaftliche Begleitforschung 2007a, ebd. S. 227.

(6) Sozialverband VdK Deutschland: Persönliches Budget; Internet http://www.vdk.de/cgi-bin/cms.cgi?ID=de16871\&SID= OdPjyBS5qp430sNMFbHymPsQLiLMZV.

(7) Wissenschaftliche Begleitforschung 2007a, ebd. S. 225.

(8) Nußbicker, Rainer (Hg.), »Ich bin jetzt Chef « Die Idee des Persönlichen Budgets in einer stationären Einrichtung für Menschen mit Behinderung, Bethel-Verlag 2007.

(9) Wissenschaftliche Begleitforschung 2007a, ebd. S. 237.

(10) Vgl. PARITÄTISCHES Kompetenzzentrum Persönliches Budget, 2 Jahre Modelle zum Persönlichen Budget für behinderte Menschen - Ein Zwischenfazit, 23.09.2006, S. 6.

(11) Z. B. Höflacher, Rainer (Sprecher der Initiative Psychiatrie-Erfahrener (Stuttgart) 2002: Stellungnahme der IPE (Stuttgart) zum »Persönlichen Budget «; Internet http://beschwerdestellepsychiatrie-stuttgart.de/ipe/Dokumente/stellungnahme_persbudget.htm

(12) Wissenschaftliche Begleitforschung 2007b: Trägerübergreifendes persönliches Budget, Zentrale Ergebnisse der Modellprojekte, S.12.

(13) So verweist z. B. der LWL in seiner Broschüre nicht auf die Trägerübergreifenden Möglichkeiten des Persönlichen Budgets.

(14) Wissenschaftliche Begleitforschung 2007a, ebd. S. 90.

(15) Ramboll Management 2008: Expertise zu Verwaltungsverfahren.

(16) BeB (2008b), Psychiatrie Jahrestagung: Jeder nach seinen individuellen Möglichkeiten. Arbeit und Selbstwirksamkeit in der Gemeinde, S. 3; Internet http://www.bebev.de/files/pdf/2008/ sonstige/2008-01-09BeBPsychiatrieJahrestagung2008.pdf.

(17) Wissenschaftliche Begleitforschung 2007a, ebd. S. 85.

(18) Wissenschaftliche Begleitforschung 2007a, ebd. S. 89.

(19) Bundesarbeitsgemeinschaft für Unterstützte Beschäftigung BAG UB: Stellungnahme Integrationsfachdienste und Persönliches Budget im Übergang Schule-Beruf; Internet http:// www.bag-ub.de/ifd/ifd_stellung_2007_01_11.pdf.

(20) BeB (2008c): Weitere Entwicklung des Persönlichen Budgets nach Abschluss des Bundesmodellprojektes, S. 7; Internet http://www.beb-ev.de/files/pdf/2008/sonstige/2008-04-12DiskussionspapierPBGL6.pdf. die individuelle Teilhabe »die dem Selbstbestimmungsrecht von Menschen mit Behinderung oder psychischer Erkrankung mehr Spielräume eröffnet und ihnen einen Platz in der Mitte der Gesellschaft sichert« (4) wesentlicher Baustein einer inklusiven Gesellschaft ist.

Die Leitidee des Persönlichen Budgets ist eng verknüpft mit diesen Ideen der gesellschaftlichen Teilhabe, der Inklusion und der individuellen und an den persönlichen Bedarfen ausgerichteten Hilfeleistung. Wie die Begleitforschung nachweist, bietet das Persönliche Budget in diesem Sinne vielfältige, erweiterte Chancen und Wahlmöglichkeiten für Menschen mit Behinderung oder psychischer Erkrankung. »Ja, mein Bewegungsfreiraum, der hat sich dermaßen erweitert durch das Budget, dass ich das wieder bezeichnen kann, voll im sozialen Mittelpunkt und Leben zu stehen. Was ja vorher nicht gegeben war.« (5) Diese Aussage eines Budgetnehmers fordert die Anbieter sozialer Unterstützungsleistung auf, nachzudenken, wie weit die bisher angebotenen Formen der Unterstützung diesen Aspekt wirklich berücksichtigt haben.

Menschen mit Behinderung oder psychischer Erkrankung gestalten, so zeigen es Aussagen von einzelnen Budgetnehmern, ihre Assistenzleistungen mit Hilfe des Persönlichen Budgets selbstbestimmt. Sie realisieren ihre individuellen persönlichen Teilhabemöglichkeiten auch jenseits der bekannten eher institutionszentrierten Unterstützungsformen. Sie erhalten darüber neue Handlungsfreiräume, da sie sich die Unterstützungsleistungen zeitlich und sozial flexibler organisieren können. Der einzelne Mensch wird so zum Kunden, Käufer oder Arbeitgeber und verfügt darüber, wann, wo, wie und von wem die Unterstützung zur Eingliederung erbracht wird. (6) »Ich kann so lange und so oft unterwegs sein, wie ich es möchte. (...) Ein besonderer Vorteil ist es bei Aktivitäten, die in die Nacht hineingehen. « (7) Diese Aussage liefert einen Hinweis darauf, dass bei einer von den Nutzern selbst organisierten Assistenz ganz neue Aushandlungsprozesse zwischen Nutzerinteressen und den ebenso berechtigten Arbeitnehmerinteressen in den Blickpunkt genommen werden müssen.

Auch die Nutzer von Persönlichen Budgets nehmen diese Chance zur Veränderung ihrer Rolle wahr, vom (nur) abhängigen Menschen mit Behinderung oder psychischer Erkrankung hin zu einem (mehr) bestimmenden Partner in der Beziehung zu ihrem jeweiligen Unterstützer. »Ich bin jetzt Chef « (8) signalisiert dieses geänderte Rollenverständnis, das gleichermaßen mit einem Anwachsen von Selbstbestimmung und autonomeren Handlungsmöglichkeiten einhergeht. Das Persönliche Budget bietet also weitere "positive (Neben-) Wirkungen, die eher das subjektive Empfinden der Budgetnehmerinnen und Budgetnehmer betreffen und auf persönliche Empowerment-Prozesse hinweisen « (9).

\section{Uneinheitliche Bedarfsfeststellung}

Bundesweit sind insgesamt mehr als 60 Instrumente zur Feststellung des Hilfebedarfes im Rahmen des Bundesmodellprojektes »entdeckt « worden. (10) Diese divergierenden Varianten der Bedarfsfeststellung führen, so zeigen es Berichte aus dem Alltag der Budgetgewährung, zu einer 
sehr unterschiedlichen Bewilligungspraxis in den einzelnen Regionen. Vor dem Hintergrund der deutlichen Entwicklung der Eingliederungshilfe in Richtung Kommunalisierung und der sich damit immer mehr an der jeweiligen Haushaltslage der Gebietskörperschaften orientierenden Auslegung der Sozialgesetzgebung gerät die Erhaltung und Sicherung einheitlicher und vergleichbarer Lebensverhältnisse für Menschen mit Behinderung oder psychischer Erkrankung in Deutschland insgesamt ins Wanken. Die Erfahrungen in den Bundesländern, die die Kommunalisierung der Eingliederungshilfe bereits umgesetzt haben, geben dafür deutliche Beispiele. Es steht nun zu befürchten, dass durch die verschiedenen Instrumente der Bedarfserhebung ebenfalls monetäre Überlegungen für die Gewährung Persönlicher Budgets (als eine Form der Hilfegewährung) in den Vordergrund treten. Daher erscheint die Forderung des Bundesverbands evangelische Behindertenhilfe ein einheitliches und an der International Classifikation of Functioning, Disability and Health (ICF) der Weltgesundheitsorganisation (WHO) orientiertes Erhebungsverfahren zu entwickeln nur sinnvoll und unabdingbar. Nur so lässt sich die Budgetbemessung für die Betroffenen nachvollziehbar und transparent darstellen und die Chance auf einen gleichwertigen Zugang zu den notwendigen Teilhabeleistungen erhalten. Dies wird von vielen Verbänden, und wesentlich auch von Betroffenenverbänden gefordert. (11)

Gleichzeitig müssen Absichten einzelner Sozialleistungsträger, das Persönliche Budget zur Kostendämpfung zu missbrauchen, in die Schranken gewiesen werden. Regelungen, die beispielsweise ambulante Leistungen grundsätzlich in einer bestimmten Höhe deckeln, sind nicht akzeptabel, da sie dem Prinzip der Bedarfsdeckung und dem Wunsch- und Wahlrecht gemäß § 9 SGB IX widersprechen. Dies ist insbesondere für die Menschen, die stationäre Einrichtungen verlassen und ambulanter Unterstützung bedürfen, ein erhebliches Handicap, schließt diese Praxis doch Menschen mit einem hohen Hilfebedarf von dieser Leistung aus.

\section{Beratung und Assistenz}

Für Menschen mit Behinderung oder psychischer Erkrankung, die einen hohen und komplexen Hilfebedarf haben, stellt sich darüber hinaus ein weiteres erhebliches Problem. Insbesondere für diese Gruppe, deren Anteil an den Budgetnehmern im Rahmen des Bundesmodellprojektes noch gering war, ist das Persönliche Budget ein eher in der längerfristigen Handhabung positiv wirkendes Programm. Menschen, die längere Zeit nicht oder vielleicht noch nie selbst über wesentliche Aspekte der Gestaltung ihrer Lebensumstände entschieden haben, können diese für sie (wieder) neue Lebenssituation nicht einfach so gestalten. Der Umgang mit wieder gewonnener Autonomie, die Einschätzung von Chancen, Gestaltungsmöglichkeiten aber auch den Risiken bedarf kontinuierlicher zusätzlicher Information, Beratung und Assistenz. Leider sind die entsprechenden Aussagen hierzu im SGB IX widersprüchlich. Während auf der einen Seite auf das Prinzip der Bedarfsdeckung hingewiesen wird, ist im gleichen Paragrafen die Bestimmung enthalten, dass die Höhe der Kosten der bisherigen Leistungserbringung nicht überschritten werden soll. Hier ist, im Interesse vor allem der Nutzerinnen und Nutzer, eine rechtliche Klärung notwendig, um für alle Beteiligten Handlungssicherheit zu schaffen und die notwendige Beratung und Budgetassistenz als zusätzliche Leistung zu finanzieren.

\section{Trägerübergreifende Leistungen}

Obwohl es in § 17 SGB IX heißt, »das Persönliche Budget wird von den beteiligten Leistungsträgern trägerübergreifend als Komplexleistung erbracht «, wurden im Projektzeitraum tatsächlich nur fünf Prozent als Trägerübergreifende Persönliche Budgets realisiert. Im Wesentlichen handelte es sich ausschließlich um Leistungen der Sozialhilfe (ca. 90\% oder 755 von 847 Persönlichen Budgets), ein Hinweis darauf, dass andere Sozialleistungen nur bedingt in den Blick genommen werden. Es spiegelt aber auch wider, dass die Sozialhilfe (Eingliederungshilfe für Menschen mit Behinderungen) die in erster Linie erreichbare Hilfe zur Eingliederung für Menschen mit Behinderung oder psychischer Erkrankung darstellt. (13) Lediglich Leistungen nach SGB XII und SGB XI (Pflegeversicherung) wurden in nennenswerterem Umfang verknüpft, nämlich 35 von 44. (14). Die geringe Nutzung der Kombination von Leistungen unterschiedlicher Sozialleistungsträger liegt sicherlich, wie bei der Pflegeversicherung, an dem sehr unattraktiven Gutscheinverfahren, das einen wirklichen Zugewinn von Wahlmöglichkeiten und Selbstbestimmung nicht unterstützt. Aber auch die nicht aufeinander abgestimmten Beantragungs- und Bewilligungsverfahren und das Fehlen einer mit ausreichenden personellen und fachlichen Ressourcen ausgestatteten unabhängigen Beratungsinstanz erschweren eine wirkliche trägerübergreifende Zusammenarbeit. Ob die vom Gutachter Ramboll Management empfohlene Stärkung der Servicestellen (15) hier eine Lösung darstellt, muss mit einer gewissen Skepsis betrachtet werden, da diese, angesiedelt bei Sozialleistungsträgern, kaum die notwendige Neutralität aufbringen können.

\section{Arbeit und Persönliches Budget}

Wenn wir davon ausgehen, dass »Arbeit bzw. Erwerbstätigkeit (...) nicht nur für die ökonomische Existenzsicherung von zentraler Bedeutung (sind), sondern auch für die soziale Identität, sozialen Kontakte sowie Selbstwirksamkeit« (16), so muss das Persönliche Budget auch für diesen Bereich wirksam werden. Von immerhin 20 Prozent der Budgetnehmerinnen und Budgetnehmer wurden Unterstützungsbedarfe im Bereich Arbeit, Beschäftigung und Bildung formuliert (17), und fast ein Drittel der Budgetnehmer war ohne Beschäftigung. Die im Projekt untersuchten Einzelfälle, in denen das Persönliche Budget für Maßnahmen zur Teilhabe am Arbeitsleben (vor allem Berufsbildung nach SGB III) genutzt wurde, machen Mut, das Persönliche Budget hier stärker zu nutzen, und vor allem für Menschen mit psychischen Erkrankungen andere, neue Formen der Teilhabe am Arbeitsleben zu kreieren.

Aber schon im Bereich der Werkstätten für Menschen mit Behinderungen (WfbM) liegen vor allem sozialversi- 
cherungsrechtliche Hindernisse (Koppelung der Sozialversicherungspflicht an die Institution WfbM) auf dem Weg zu einer Verwirklichung Persönlicher Budgets. Auch die Anforderung der Integration in den ersten Arbeitsmarkt (§ 136 SGB IX) steht unverbunden neben öffentlich subventionierten Anreizen zum Verbleib in der Werkstatt.

Auf der anderen Seite aber stellt das Persönliche Budget für Menschen mit Behinderung oder psychischer Erkrankung »gerade hinsichtlich des Übergangs in Beschäftigungsverhältnisse jenseits der Werkstätten für behinderte Menschen eine große Chance dar. Mit Hilfe des Persönlichen Budgets sind individuell ausgerichtete Lösungen in einer Flexibilität möglich (z. B. durch Arbeitsbegleitung und Job Coaching), die bisher aufgrund der institutionell vorgegebenen Rahmenbedingungen nicht denkbar waren« (19), so die Bundesarbeitsgemeinschaft für Unterstützte Beschäftigung (BAG UB) in einer Stellungnahme.

\section{Dynamik nutzen}

Noch ist die Ausgestaltung des Persönlichen Budgets nur bedingt gelungen. Noch überwiegen in der Einschätzung, neben den nicht gering zu bewertenden geschilderten subjektiven Verbesserungen, die eher institutionell und strukturell vorhandenen Verbesserungsbedarfe.

Dennoch, das Persönliche Budget befördert in einer ganz besonderen Weise den paradigmatischen Wandel von der Fürsorge zur Dienstleistung, der von allen am Hilfeprozess Beteiligten langfristige Lern- und Veränderungsprozesse erfordert (20), wie es der Bundesverband evangelische Behindertenhilfe in seinem Diskussionspapier formuliert. Diese dem Persönlichen Budget innewohnende Dynamik muss auf andere Bereiche der Assistenzen für Menschen mit Behinderung oder psychischer Erkrankung ausstrahlen.

\section{Resümee}

Dabei geht kein Weg an einer konsequenten Teilhabeorientierung vorbei, die Maßstab für alle Unterstützung von Menschen mit Behinderung oder psychischer Erkrankung sein muss. Dieser Grundsatz gilt daher nicht nur für die als Persönliches Budget erbrachten Leistungen, die ganz selbstverständlich eigenständig von den Nutzerinnen und Nutzer gestaltet werden. Auch im Bereich der Sachleistung muss dies Prinzip konsequenter als bisher Einzug halten. Wenn Einrichtungen und Dienste ihre Struktur, Arbeitsorganisation und ihre Angebote darauf ausrichten, so wird die individuelle Teilhabe zum zentralen Maßstab für die Qualität der professionell erbrachten Unterstützung. Eine solche Veränderung schafft mehr Raum für individuelle und auf den Einzelnen bezogene Unterstützung.

Die Nutzerinnen und Nutzer werden dabei von den Profis deutlich als mitwirkende Partnerinnen und Partner gebraucht. Dies vor allem in der Gestaltung der alltäglichen individuellen Begleitung in allen Lebenslagen.

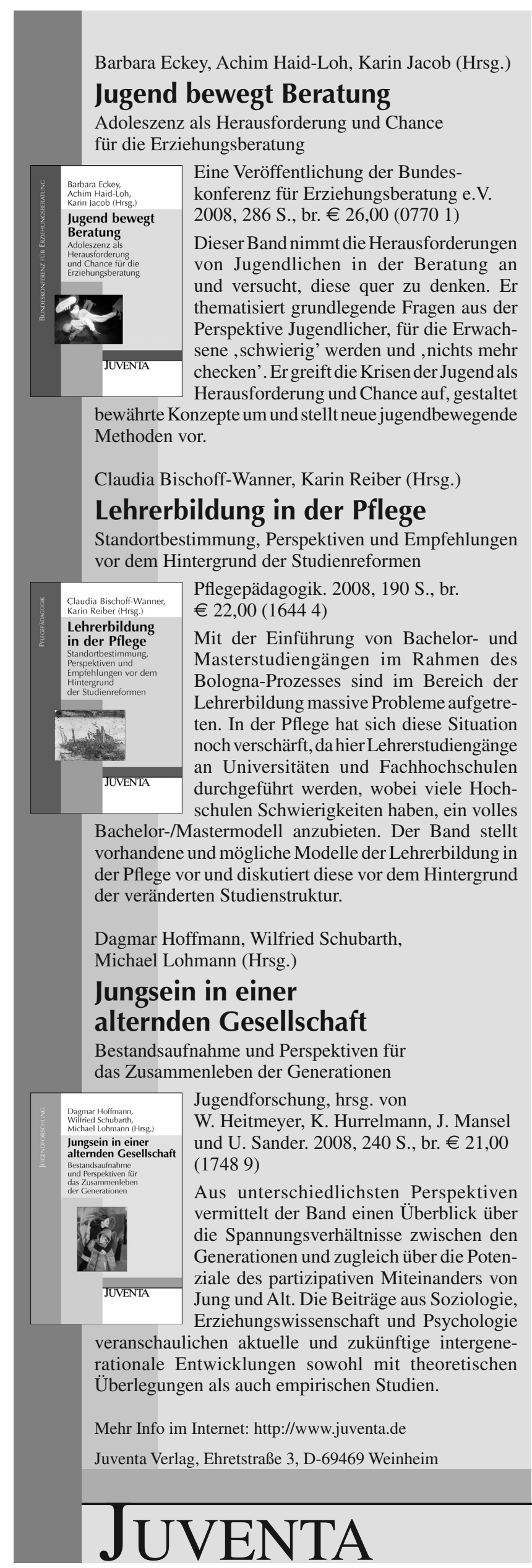

